\title{
Correlative Fluorescence and Scanning Electron Microscope Imaging of Cultured Neurons Pretreated with Ionic Liquid
}

\author{
Atsushi Muto ${ }^{1}$, Mikihiro Shibata ${ }^{2}$, Mami Konomi $^{3}$, Ryohei Yasuda ${ }^{2}$ and Naomi Kamasawa ${ }^{2}$ \\ 1. Nanotechnology Systems Div., Hitachi High Technologies America, Clarksburg, MD, USA \\ 2. Max Planck Florida Institute for Neuroscience, Jupiter, FL, USA \\ 3. Hitachi High-Technologies, 1-24-14, Nishi-shimbashi, Minato-ku, Tokyo, Japan
}

Ionic liquids are organic salts that are liquid at room temperatures; some are non-volatile and have high ionic conductivity. HILEM IL1000 (Hitachi High-Technologies) is an ionic liquid designed for use in electron microscopy (Fig. 1) [1]. It is stable under vacuum, conductive, hydrophilic and hyperosmotic, while also being inert and chemically safe [2]. Furthermore, it has the unique capability to maintain wet samples in a "hydrated" state under vacuum conditions. IL1000 has been applied to biological samples including bacteria, yeast, fungi and hard-shelled organisms such as worms and shrimp for successful imaging of fine morphologies [3, 4]. We now report the application of IL1000 to the traditionally difficult process of SEM imaging of neuronal cultured cells.

SEM imaging of neuronal cells grown on glass or plastic substrates is challenging because the cells contain a large amount of water within delicate cell membranes. In addition, the somas of pyramidal neurons are relatively large (approx. $20 \mu \mathrm{m}$ diameter) and axons and dendrites extend widely. Shrinkage and deformation of these structures due to conventional SEM sample preparation methods is common. In this study, IL1000 was used to observe cultured neurons without the conventional dehydration process to correspond with live images captured by high-speed AFM [5].

Dissociated hippocampal neurons were cultured on poly-L-ornithine-coated Thermanox coverslips (Thermo Scientific) in a medium at $37 \mathrm{C}$ in $5 \% \mathrm{CO}_{2}$ [3]. Cells were fixed at 21 days in vitro with paraformaldehyde or glutaraldehyde in $0.1 \mathrm{M}$ phosphate buffer $(\mathrm{pH} 7.4)$ for 2 hours. After washing with the same buffer, $5 \%(\mathrm{w} / \mathrm{v})$ IL1000 was dropped on the fixed neurons to totally cover whole plastic coverslips, and the samples were kept in an ambient temperature for $4 \mathrm{~h}$ or overnight. The coverslips were lightly washed with distilled water to remove excess liquid on cell surface and air-dried. The samples were examined in a confocal laser microscope and then observed by Hitachi SU8200 scanning electron microscope at $1-1.5 \mathrm{kV}$ acceleration voltage with or without light Pt coating.

The IL1000 pretreated cells were imaged with minimum charging at $1.5 \mathrm{kV}$ using a lower SE detector. A pyramidal shape of the cell body was maintained without deformation under high vacuum conditions (Fig. 2). Dendrites extended from the cell body were also clearly observed and followed to the proximal tips. The surface of the dendrites contained many filopodia and small protrusions in contact with neighboring neurites (Fig. 2b). Adhesion onto the coverslip looked intact compared to the sample prepared by a conventional dehydration method. No significant difference was seen in cells fixed with only paraformaldehyde versus glutaraldehyde. The usage of IL1000 for a correlative fluorescence and electron microscopy was demonstrated via a newly developed workflow of overlaying a fluorescence image onto a live SEM image. A fluorescence image and its corresponding SEM image of cultured neurons are shown in Figure 3. GFP fluorescence was nicely preserved post IL1000 treatment. 


\section{References:}

[1] E.Nakazawa et al., Proc. of the Fifty-sixth Symposium of the Japanese Society of Microscope., 47-2, p92-95 (2013)

[2] K. Kawai et al., Langmuir, 27, 7353-7356(2011)

[3] M. Sakaue et al., Microsc. Microanal. 20 (Suppl. 3) , pp 1012-1013(2014)

[4] S. Shiono et al., Microsc. Microanal. 20 (Suppl. 3), pp 1016-1017(2014)

[5] M. Shibata et al,.Sci.Rep.5, 8724 DOI:10, 1038,srep/07824(2015)

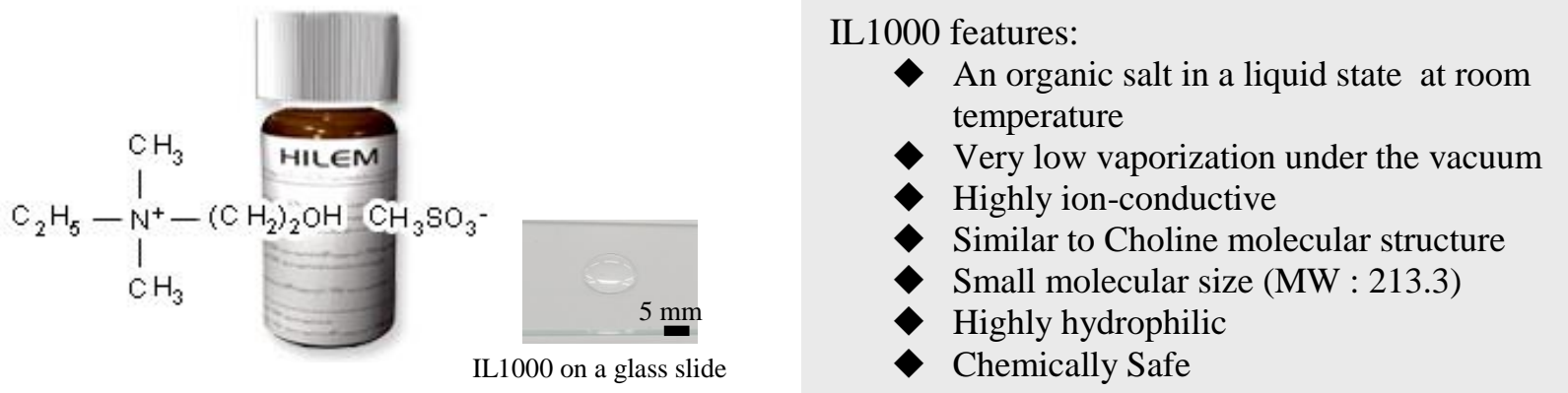

Fig.1. Ionic Liquid IL1000 outlook and features
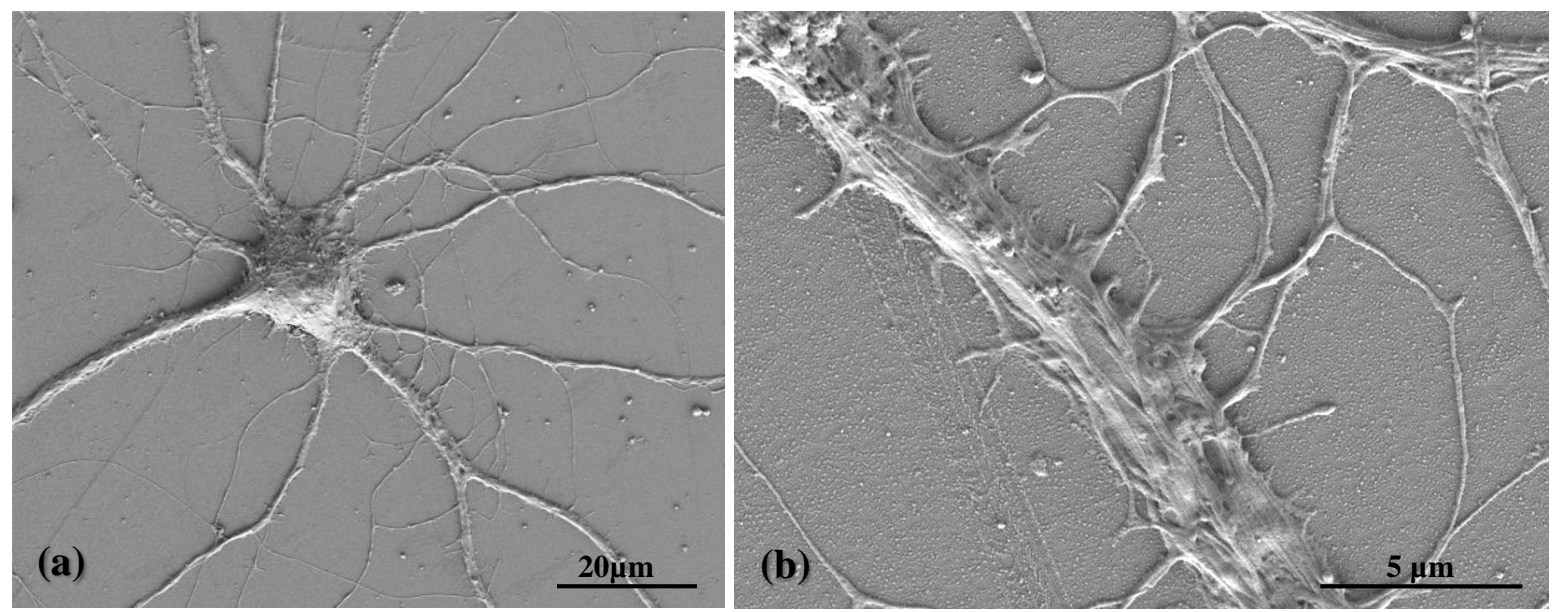

Fig. 2. SEM images of a cultured neuron on a coverslip treated in ionic liquid

(a) Whole cell view

(b) Magnified image of dendrites. Note many filopodia and small protrusions are not detached from the substrate.
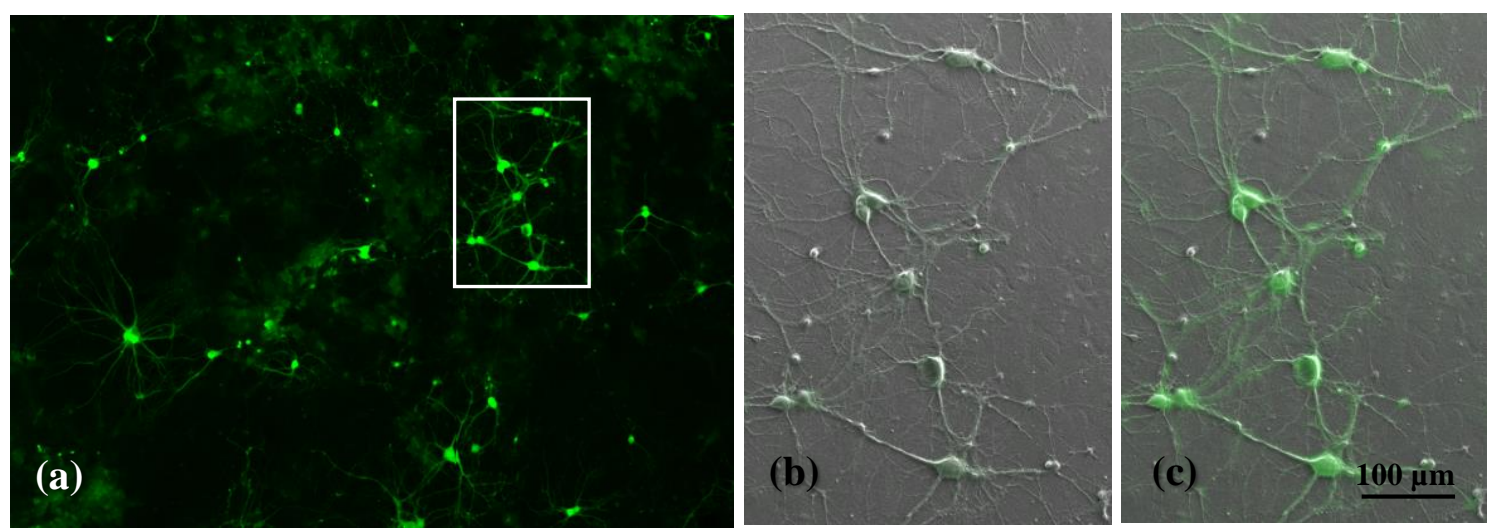

Fig. 3. Fluorescence and SEM images of a cultured neuron on a coverslip treated in ionic liquid.

(a) GFP fluorescence image

(b) SEM image of boxed area on GFP fluorescence image

(c) Correlative image of GFP fluorescence and SEM image 